\title{
Photophysical Properties of CdSe/CdS core/shell Quantum Dots with Tunable Surface Composition
}

Kelly L. Sowers ${ }^{1}$, Zhentao Hou ${ }^{1}$, Jeffrey J. Peterson ${ }^{1}$, Brett Swartz ${ }^{1}$,Sougata Pal, ${ }^{3}$ Oleg Prezhdo ${ }^{3}$, and Todd

D. $\operatorname{Krauss}^{1,2, *}$

Department of Chemistry ${ }^{1}$ and the Institute of Optics ${ }^{2}$, University of Rochester, Rochester, NY 14627

Department of Chemistry, University of Southern California, Los Angeles, CA $90089^{3}$

\begin{abstract}
We report the synthesis and optical characterization of core/shell CdSe/CdS quantum dots (QDs) with controlled surface composition. Using secondary phosphine chalcogenide and cadmium carboxylate precursors in an alternating layer-by-layer synthetic approach, the elemental surface composition of the quasi-type-I CdSe/CdS core/shell QDs can be repeatedly tuned from predominately cadmium to predominantly sulfur, as measured by X-ray photoelectron spectroscopy (XPS). Similar to CdS and CdSe core-only QDs, the surface composition has a significant effect on the photoluminescence (PL) quantum yield: sulfur terminated QDs exhibit quenched PL, while cadmium terminated QDs have relatively bright PL. Density-functional tight-binding calculations on $\mathrm{CdSe} / \mathrm{CdS}$ core/shell clusters suggest that PL quenching for sulfur-rich surfaces is the result of a high density of hole surface states in the QD bandgap. Time-resolved PL measurements confirm the QDs' nonradiative recombination rates are strongly sensitive to the surface composition.

*krauss@chem.rochester.edu
\end{abstract}




\section{Introduction}

Colloidal semiconductor nanocrystals, commonly known as quantum dots (QDs), have been the source of much study over the past two decades, owing to a wide range of customizable features including size-dependent optical properties ${ }^{1-3}$. Controlled modifications of surface composition and surface passivation can be used to tune QD photophysical properties, such as photoluminescence energy and intensity ${ }^{4-7}$. Since particle size, composition, and surface passivation can be controlled in the initial QD synthesis reaction ${ }^{8}$ and post-synthetic workup, QDs can potentially be useful in a wide range of applications such as light harvesting for solar fuels ${ }^{9,10}$ and solar cells ${ }^{11,12}$, displays and LEDs ${ }^{13,14}$, and biological imaging ${ }^{15}$, labeling ${ }^{16}$, and cell stimulation ${ }^{17}$.

Due to their large surface to volume ratio, the surface of QDs has been historically recognized as integral in influencing the optical properties of colloidal QDs ${ }^{6,18-26}$. For example, early synthetic breakthroughs that produced highly-fluorescent QDs were enabled, in part, by the discovery that phosphine ligands provided effective passivation of dangling bonds at the nanoparticle surface ${ }^{27}$. Subsequent development of core/shell structures (e.g. CdSe/ZnS), dramatically enhanced QD photoluminescence (PL), PL quantum yield (QY), and particle photostability ${ }^{24,28}$. Such efforts significantly advanced the potential for QDs in various technological applications and helped enable new levels of understanding in QD photophysics. Current research seeks even greater tunability and optimization of QD optical properties through surface engineering ${ }^{29,}{ }^{30}$, such as synthesis of "giant" shells ${ }^{31,32}$ and alloyed QD cores and shells $s^{33-35}$ to minimize single particle PL blinking. Photophysical properties can be tuned through wave function engineering provided by type-I and type-II heterostructures ${ }^{36-38}$, and metallo-ligands to tune PL over broad spectral regions $s^{39,40}$.

One historical challenge in engineering the QD surface has been achieving precise compositional control of the surface chemistry. For metal chalcogenide QDs, such control is difficult due to a limited understanding of the specific molecular reactive species of metal and chalcogenide precursors, and the 
mechanisms involved in QD nucleation and growth. However, recent reports suggest that by employing secondary phosphine sulfide precursors in colloidal QD synthesis (such as diphenyl phoshine sulfide $(D P P S))^{41,42}$ the surface composition can be tuned from all metal to all sulfur. Furthermore, the composition of a QD surface can also have dramatic effects on the QD optical properties; for core-only CdSe or $\mathrm{CdS}^{43} \mathrm{QDs}$, when the surface is cadmium terminated the QD exhibits bright PL, however when the surface is S- or Se-terminated the PL is quenched. These effects are believed to originate from midgap hole states that are associated with surface $\mathrm{S}$ or Se atoms and that effectively trap photoexcited holes $^{43}$.

We sought to extend these studies to core/shell CdSe/CdS QD heterostructures and investigate the effects of surface composition on their photophysical properties. In particular, such studies can provide information about the relative extent of the electron and hole wavefunctions in these nanoparticles. The simplest thinking suggests that due to the relative energy band offsets for CdSe and CdS, CdSe/CdS core/shell QDs are expected to localize the hole wavefunction in the core of the particle $e^{44,45}$. If holes are limited in sampling the surface in a core/shell QD and the surface is the origin of the PL quenching, it is possible that changing QD surface chemistry will not significantly affect PL. Contrarily, the presence of a significant number of hole energy states in the bandgap for CdS could drive the hole toward localization on the CdSe/CdS QD surface, even though it would have to tunnel through a small energy barrier $(\sim 0.88 \mathrm{eV})^{46}$ due to the CdS layer. In this case, the PL intensity should rise and fall with cadmium or sulfur QD termination.

Here, we describe the synthesis of core/shell CdSe/CdS QDs with controlled surface composition using exclusively secondary phosphine chalcogenide presursors. By adopting an alternating layer-bylayer synthetic approach ${ }^{47}$, the surface composition of the CdSe/CdS QD can be reversibly tuned from predominantly cadmium to predominantly sulfur ${ }^{43}$, as verified with $\mathrm{X}$-ray photoelectron spectroscopy (XPS). Similar to previous studies on bare core QDs ${ }^{43,48}$, the surface composition is shown to have a 
significant effect on the PL QY. Sulfur terminated QDs have dramatically quenched PL and longer fluorescence lifetimes, while cadmium terminated QDs have significantly brighter PL and shorter fluorescence lifetimes. Density-functional tight-binding (DFTB) calculations on CdSe/CdS QDs suggest the presence of a high density of hole surface states in the QD bandgap quenches PL, despite the quasi-typeI electronic heterostructure thought to effectively trap the hole in the core.

\section{Methods}

CdSe QD Synthesis. CdSe QDs were synthesized using adaptations of an air-free, hot injection method as previously reported $^{49} .1 .0 \mathrm{mmol}$ of diphenylphosphine selenide (DPPSe; $0.266 \mathrm{~g}$ ) was dissolved in a small volume of toluene $(\sim 1-3 \mathrm{~mL})$ at $60{ }^{\circ} \mathrm{C}$ in an inert atmosphere. Concurrently, in a separate reaction flask, $3.0 \mathrm{mmol}$ of $0.2 \mathrm{M}$ cadmium oleate $(\mathrm{Cd}($ oleate $) 2 ; 15 \mathrm{~mL}$ ) and a small volume of 1-octadecene (ODE; 10 $\mathrm{mL}$; degassed under vacuum overnight at room temperature) were heated to $270{ }^{\circ} \mathrm{C}$. At temperature, the DPPSe/toluene mixture was rapidly injected and the temperature was dropped to $220{ }^{\circ} \mathrm{C}$. Small aliquots (0.1 to $0.5 \mathrm{~mL}$ ) were removed every minute and growth was monitored by UV-visible spectrophotometry. QDs were allowed to continue growing at $220^{\circ} \mathrm{C}$ until the first exciton absorbance peak ceased shifting, approximately 2-5 minutes. When QD growth had ceased, the reaction was quenched by removing the flask from heat and applying a cool air flow to the outside of the flask. The resulting QD product was kept under a positive nitrogen flow for the shelling reactions.

CdS/CdSe core/shell QD Synthesis. CdS shells were grown using variations of a successive ionic layer absorption and reaction (SILAR) method ${ }^{47}$. Volumes of cadmium and sulfur precursors for each full monolayer were calculated for a given diameter ${ }^{50}$ and surface area of the core QD, accounting for increased surface area with each subsequent shell. As-prepared CdSe QD cores were kept in a flask under continuous nitrogen flow during the shelling procedure, at a temperature of $235^{\circ} \mathrm{C}$. All shelling reactions began by introducing a layer of cadmium to the CdSe cores through swift injection of $\mathrm{Cd}$ (oleate) $)_{2}$ at $235{ }^{\circ} \mathrm{C}$, and reacting for 15 minutes to produce cadmium terminated CdSe-QDs. We 
denote such species as CdSe-Cd QDs. In the same flask, a monolayer of sulfur was grown by promptly injecting either a diphenylphosphine sulfide (DPPS) or di-isobutylphosphine sulfide (DiBPS) solution, and reacting for 15 minutes to produce sulfur terminated CdSe-CdS (sample A), completing the first full shell. Subsequent injections were performed, alternating $\mathrm{Cd}$ and $\mathrm{S}$ until 3 full shells were grown (samples B-E), followed by an additional cadmium layer, producing QDs with the composition CdSe-CdS/CdS/CdS/Cd (sample F). Fractions of core-shell QDs were withdrawn (0.1-0.5 mL aliquots) at the end of each 15 minute reaction time (before the next injection) and diluted with hexanes. Sulfur terminated QDs synthesized using DPPS were observed to rapidly agglomerate and precipitate in hexanes, whereas the same cadmium terminated QD fractions were stable. In contrast, both sulfur and cadmium terminated QDs synthesized using DiBPS formed a stable colloid in hexanes.

Optical characterization. Absorbance data was taken using a Perkin-Elmer Lambda 35 UV/Vis spectrophotometer, and PL data were taken on a modular Acton fluorometer system. Samples were dissolved in hexanes and placed in a $1 \mathrm{~cm}$ square quartz cuvette. Optical densities were controlled to be between 0.01 and 0.1 .

Fraction preparation. In preparation for electron microscopy (TEM) and XPS experiments, aliquots in hexanes were flocculated using a 1:4:1:1:3 ratio of QDs(hexanes) : acetone : ethanol : butanol : methanol. Samples were centrifuged, the supernatant discarded, and the resultant QD pellet was resuspended in hexanes.

Transmission Electron Microscopy. TEM images were taken with an FEI Tecnai F-20 field emission electron microscope, operated with an accelerating voltage of $200 \mathrm{keV}$. Samples were prepared as above, then dropcast onto ultrathin carbon film grids for larger QDs, or silicon nitride grids for smaller QDs. 
X-ray Photoelectron Spectroscopy (XPS). XPS data were taken with AI Ka as the source of incident X-rays. QDs were purified as described for the TEM measurements above. Thick QD films were drop cast on $\mathrm{O}_{2}$ plasma-cleaned silicon wafers for XPS measurements. Gaussian functions were used to fit XPS data individually, to determine relative signals from surface or inner atoms. Due to the unique electron binding energies of $\mathrm{Cd} 3 d \mathrm{~d} / 2$ and $3 / 2$ (approximately 405 and $412 \mathrm{eV}$ respectively) and $\mathrm{S} 2 \mathrm{p} 3 / 2$ and $1 / 2$ (approximately 162 and $163 \mathrm{eV}$ respectively) ${ }^{51}$, the relative chemical composition of the QD can be determined straightforwardly assuming a spherical QD with a core radius determined from the first exciton absorption ${ }^{50}$. Subsequent shell radii can be extrapolated by the addition of unit cell dimensions of the CdS crystal ${ }^{52}$ as confirmed by TEM analysis (Figure S2). The Cd-to-S ratios were obtained by XPS survey scans and corrected for atomic sensitivity factors ${ }^{48,53,54}$. If the amount of $\mathrm{Cd}$ or $\mathrm{S}$ atoms in a QD sample is known, based on particle size the numbers of surface atoms for $\mathrm{Cd}$ or $\mathrm{S}$ can be determined from the integrated elemental region signals from the surface versus interior XPS peaks ${ }^{43}{ }^{48}$. By comparing the total atoms of $\mathrm{Cd}$ or $\mathrm{S}$ to the percent of surface atoms, relative surface composition can be inferred.

PL Lifetime Measurements. A drop (approximately $6 \mu \mathrm{L}$ ) of the $\mathrm{QD}$ solution in 1-octadecene was deposited onto a glass coverslip. A 488-nm excitation beam from a picosecond pulsed diode laser (PicoQuant PDL $800-D$ ) filtered by a $482 / 18 \mathrm{~nm}$ band pass filter and circularly polarized by a quarterwave plate was reflected by a $488-\mathrm{nm}$ dichroic mirror to a Nikon $10 x 0.30$ N.A. Plan Fluor objective. The emission coming out of the microscope (Nikon TE-2000) passed through a 100- $\mu \mathrm{m}$ pinhole and a 488-nm long pass filter, and imaged on an avalanche photodiode (Micro Photon Devices PDM Series 5CTB) by a pair of lenses. Photon statistics were acquired with a PicoHarp 300 Time-Correlated Single Photon Counting (TCSPC) module to construct the fluorescence intensity trajectory over many cycles. Fluorescence lifetimes were determined by fitting the TCSPC data with a double exponential function. 
Amplitude-weighted lifetimes were calculated using the $\tau=\sum_{i} A_{i} \tau_{i}$, where $\tau$ is the amplitude-weighted lifetime, $\tau_{i}$ are the lifetime components, and $A_{i}$ are their corresponding normalized coefficients.

Computational methodology: To elucidate the role of composition on the PL intensity, we performed self-consistent charge density-functional tight-binding (SCC-DFTB) calculations. The SCC-DFTB method is based on a second-order expansion of the total Kohn-Sham energy in density-functional theory (DFT) with respect to charge density fluctuations. The one and two center matrix elements of the associated Hamiltonian are calculated in an explicit minimal valence basis, where the repulsive energy part is approximated by universal pair potentials, which are fitted using the data of self-consistent field calculations. The SCC-DFTB has a minimal number of input parameters by retaining the efficiency of TB approaches and has been described in detail elsewhere ${ }^{55-58}$. This work uses the SCC-DFTB parameter set, developed recently and extensively tested for cadmium-chalcogenide systems ${ }^{59}$.

\section{Results and Discussion}

X-ray Photoelectron Spectroscopy Analysis of Particle Surface Termination

X-Ray photoelectron spectroscopy (XPS) data from a series of CdSe/CdS core/shell QDs with alternating Cd or S surface passivation is shown in Figure 1. XPS is a widely-used technique for providing information about the surface composition of solid state materials ${ }^{53}, 54$, due to the slightly higher electron binding energies of surface atoms compared to respective bulk values ${ }^{60}$. Peaks assigned to interior atoms of $\mathrm{Cd} 3 d 5 / 2$ and $3 / 2$ and $S 2 p 3 / 2$ and $1 / 2$ electrons are observed to occur at 405 and 412 $\mathrm{eV}$ and 162 and $163 \mathrm{eV}$ respectively ${ }^{43}$, ${ }^{2}$. Notably, for each interior peak there exists a peak shifted approximately $1.5 \mathrm{eV}$ to higher energies due to contributions from surface atoms ${ }^{60}$. Generally speaking, the SILAR process worked as expected: QDs that had a terminal layer of $S$ applied had a strong XPS signal

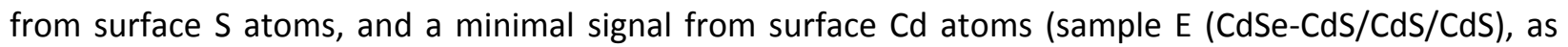
shown in Figure 1a. The opposite was also observed, cadmium terminated surfaces showed strong XPS 
data from primarily surface $\mathrm{Cd}$, and not $\mathrm{S}$ atoms (sample $\mathrm{F}(\mathrm{CdSe}-\mathrm{CdS} / \mathrm{CdS} / \mathrm{CdS} / \mathrm{Cd}$ ) as shown in Figure $1 b$.

Quantitative analysis of the XPS data yielded the relative amount of cadmium or sulfur atoms on the surface of the QD as described in Table 1. The XPS data reveals that surfaces nominally terminated with sulfur have a high percentage of surface sulfur atoms ( $>70 \% \mathrm{~S}$ for all cases). While the $\mathrm{Cd}$ terminated surfaces do show a preponderance of surface $\mathrm{Cd}$ atoms $(60-70 \% \mathrm{Cd})$, the fraction is not nearly as great as for the sulfur termination. This latter result is in contrast to small, similarly prepared CdS QDs (i.e. using secondary phosphine sulfur and Cd-carboxylate precursors), which showed greater than $90 \%$ of the surface atoms are $C d$ for a cadmium terminated $C d S Q D^{43}$. One source of error that could possibly account for the difference in the XPS data may be due to the size of the QD. For CdSe/CdS QDs, Cd atoms are located throughout the QD down to the very core. The attenuation of the XPS signals for interior atoms, and variable escape depths for different types of atoms are accounted for through an atomic sensitivity factor of the instrument, and by additional calibration to account for the differences in electron mean free paths of $C d$ and $S^{61,62}$. For these experiments, the inelastic mean free paths $(\lambda)$ were calculated to be $\sim 19.8 \AA$ for $\mathrm{Cd}$, and $\sim 23.3 \AA$ for S. Thus, in the analysis for larger QDs (i.e. the ones with thicker shells where the Cd surface percentage seems "low") the interior Cd atom XPS signal may not accurately reflect the true amount of $\mathrm{Cd}$ present. Another possible explanation is that the $\mathrm{Cd}$ or $\mathrm{S}$ precursors, although added sequentially, do not react to completion and addition of the other element increases the number of stoichiometric CdS units on the surface. Finally, the increasingly non-spherical particle morphology with increasing shell thickness (Figure S2) will also contribute to uncertainties in the quantitative analysis of the surface atom percentage. Particle size analysis of TEM images shown in Figure S2 shows agreement with predicted average particle size as determined by core size and CdS lattice parameters. While shelled QDs do demonstrate less uniform shapes as the shell thickness increases, this non-uniformity will result in only small perturbations to PL energies or linewidths, as 

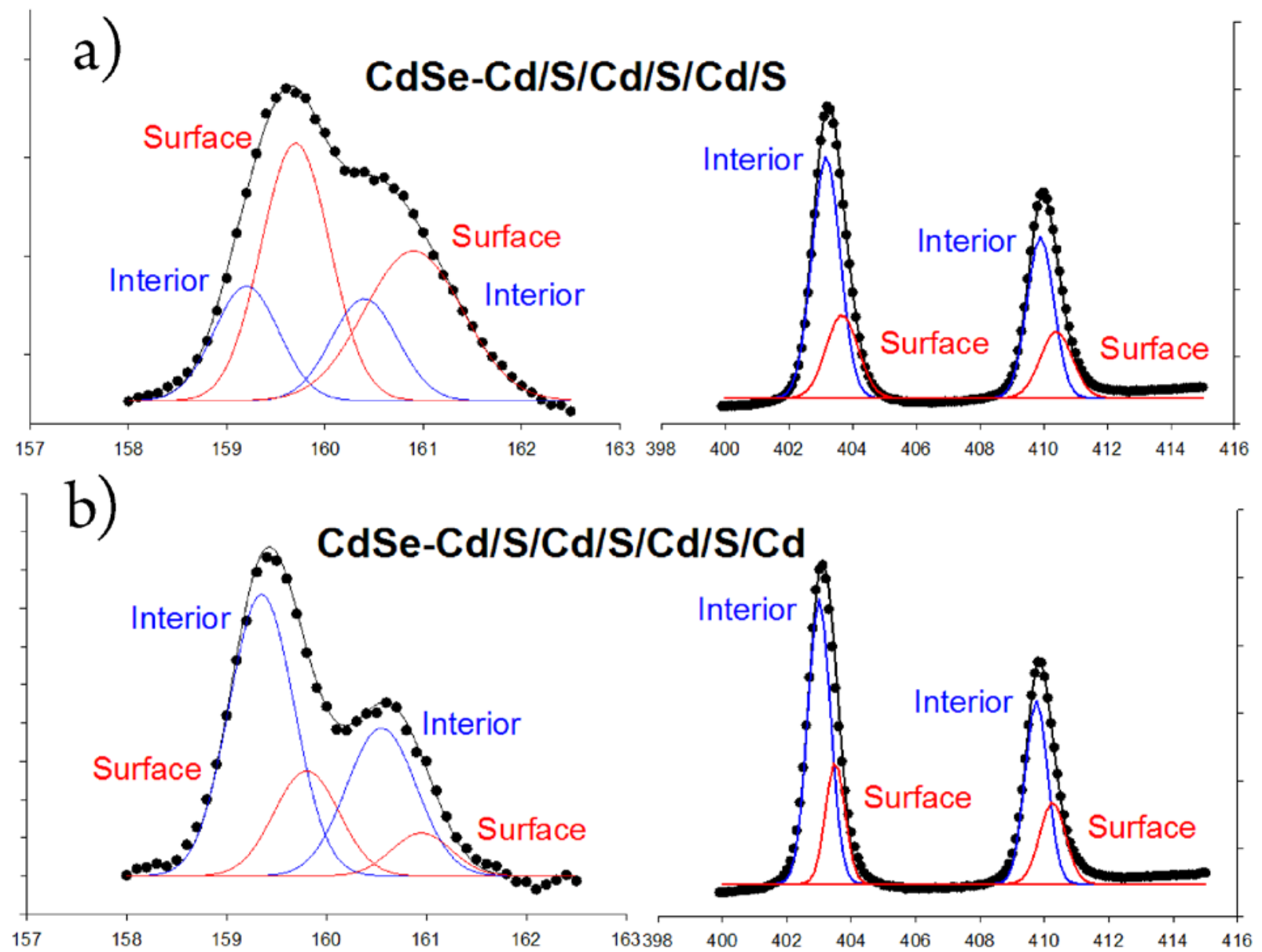

Figure 1: XPS elemental analysis of two core/shell samples: a) CdSe/CdS/CdS/CdS (sample E), and b) $\mathrm{CdSe} / \mathrm{CdS} / \mathrm{CdS} / \mathrm{CdS} / \mathrm{Cd}$ (sample F). XPS spectra corresponding to $\mathrm{Cd}$ and $\mathrm{S}$ signals are shown on the left and right, respectively. Black dots represent raw XPS data, black lines are total summed Gaussian curve fits, blue lines are Gaussian curve fits corresponding to interior atoms, and red lines are Gaussian curve fits corresponding to the higher-energy offsets attributed to surface atoms.

\begin{tabular}{ccccccccccr} 
Sample & Cd\% & Se\% & S\% & Cd/S & Corrected & $\begin{array}{c}\text { D } \\
(\mathrm{nm})\end{array}$ & $\mathrm{Cd}_{s} / \mathrm{Cd}_{\mathbf{i}}$ & $\mathrm{S}_{\mathrm{s}} / \mathrm{S}_{\mathbf{i}}$ & \multicolumn{2}{c}{ Surface \% } \\
A & 5.15 & 0.18 & 4.01 & 1.28 & 1.10 & 3.0 & 0.21 & 2.42 & 21 & 79 \\
B & 8.66 & 0.20 & 6.57 & 1.32 & 1.14 & 3.3 & 0.75 & 0.24 & 72 & 28 \\
C & 9.04 & 0.26 & 8.35 & 1.08 & 0.93 & 3.7 & 0.16 & 2.14 & 16 & 84 \\
D & 9.50 & 0.21 & 6.45 & 1.47 & 1.27 & 4.0 & 0.44 & 0.35 & 60 & 40
\end{tabular}




$\begin{array}{lllllllllll}\text { E } & 16.61 & 0.38 & 16.72 & 0.99 & 0.85 & 4.4 & 0.43 & 2.28 & 27 & 73 \\ \text { F } & 8.98 & 0.17 & 6.66 & 1.35 & 1.17 & 4.7 & 0.42 & 0.33 & 58 & 42\end{array}$

Table 1. XPS compositional analysis of samples A-F.

Effects of Surface Composition on Photoluminescence

The specific surface composition of $\mathrm{CdSe} / \mathrm{CdS}$ core/shell QDs has a significant impact on the photoluminescence quantum yield (QY), as shown in Figure 2. When the QD is cadmium-terminated, strong PL is observed; when the QD is sulfur-terminated, the PL is quenched (Table S1). The quenching of PL with sulfur is a reversible effect: application of $\mathrm{Cd}$ to a sulfur terminated surface results in fluorescence recovery, while subsequent application of $\mathrm{S}$ to the cadmium terminated surface quenches the PL (Figure 2). Control experiments showed that the quenched PL from the sulfur-terminated QDs is not due to a visible degradation of the structural or optical absorbance properties of the QDs, as evidenced by absorbance (Figure S1) and transmission electron microscopy (TEM) (Figure S2) data. Additionally, this reversible PL quenching effect does not depend on the specific identity of secondary phosphine sulfide: di-isobutyl phosphine sulfide (DibPS) and diphenyl phosphine sulfide (DPPS) showed similar results (Table S1).

This alternating bright-quenched PL behavior with alternating $\mathrm{Cd}$ or S layers is very similar to that observed in $\mathrm{CdS}^{43}$ and $\mathrm{CdSe}^{48}$ core-only QDs. For CdS core-only QDs, the quenching of the PL with sulfur termination was hypothesized to arise from an abundance of hole-trap states in the energy gap, similar to the effect of capping QDs with small molecules containing thiol groups ${ }^{18,63,64}$. Interestingly, it was recently shown that thiol capped CdSe/CdS QDs could have a QY $>30 \%$, which is extremely high, ${ }^{65}$ and may at first contradict the idea that sulfur terminated QDs have quenched PL. However, even for these CdSe/CdS QDs, which were of extremely high quality, exchanging the native phosphine caps with PEGylated-thiol ligands resulted in significant PL quenching. ${ }^{65}$ Some PL quenching was attributed to imperfect surface passivation, ${ }^{65}$ which must also be considered as a source of quenching in our case as 
well. However, for CdS QDs, DFT calculations clearly showed the appearance of mid-gap states in sulfurrich particles, which act as trap states to quench PL. We suspected mid-gap trap states could similarly act to quench the PL in CdSe/CdS core/shell QDs. Further, it is possible that the formation of mid-gap states due to the sulfur rich surface also played a role in the PL quenching for the CdSe/CdS thiol capped QDs, and that a higher fraction of surface sulfur would have resulted in QDs displaying even more quenching. DFT calculations were performed to quantify the presence of such trap states in these particles.

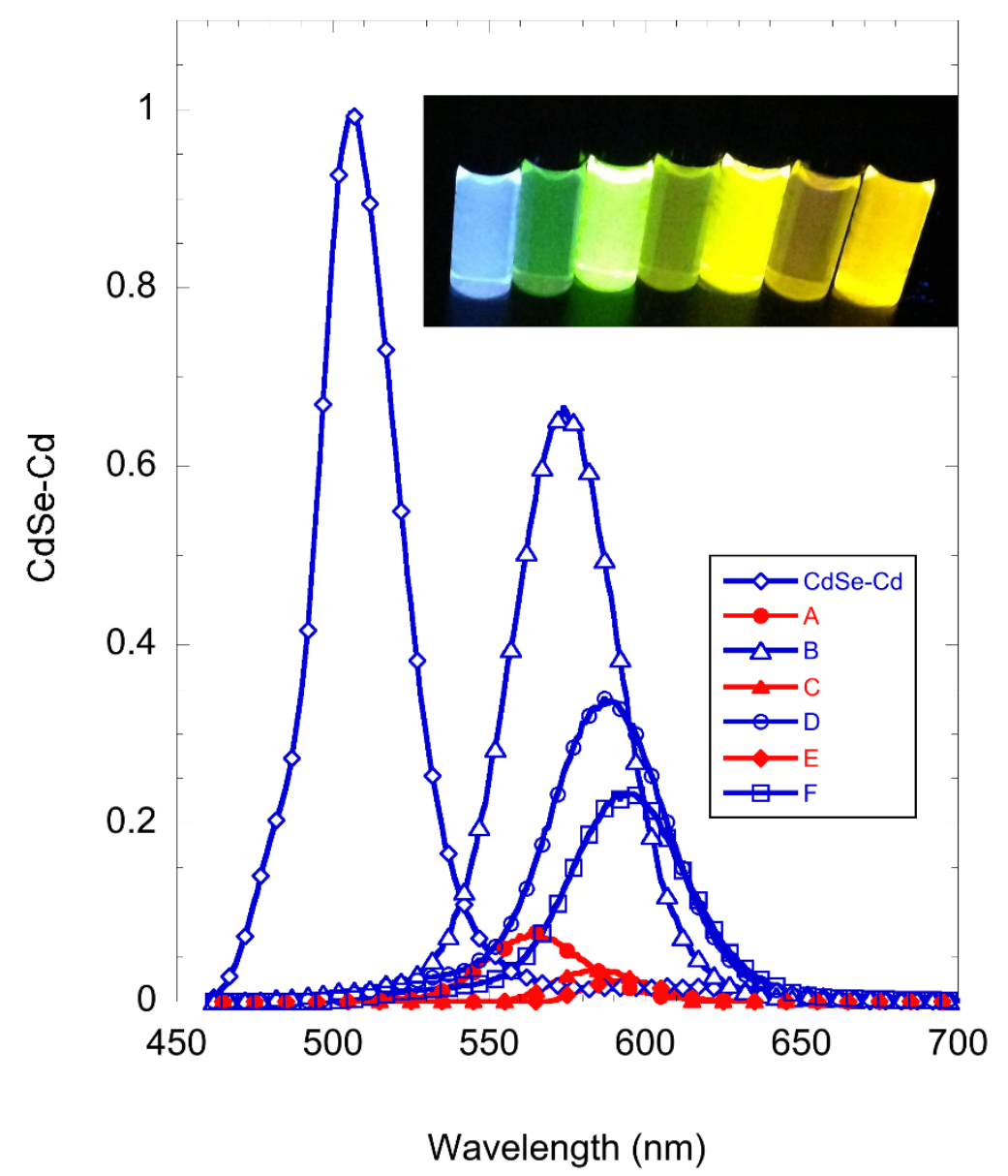

Figure 2. (Main) Photoluminescence spectra of a set of shelled QDs, normalized to each sample's individual absorbance at the excitation wavelength to account for variations in concentration. Samples are CdSe-Cd, A, B, C, D, E, and F as indicated in the legend. In this particular example, CdSe-Cd had the largest QY, while for other batches sample B was found to be the brightest of the set. (Inset) Photograph of the same QDs, of similar optical density, illuminated under UV lamp, demonstrating the visibly quenched and subsequently restored fluorescence within a single reaction. 
Calculations of CdSe/CdS Surface Trap States

To represent CdSe/CdS core/shell QDs with a nanostructure that is computationally tractable, we chose a fixed core size of $\mathrm{Cd}_{17} \mathrm{Se}_{17}$, and a shell with fixed thickness and variable surface composition of $\mathrm{Cd}_{39+/-}$ ${ }_{x} \mathrm{~S}_{39-/+x}$. First, the stoichiometric core/shell QD $\mathrm{Cd}_{17} \mathrm{Se}_{17} / \mathrm{Cd}_{39} \mathrm{~S}_{39}$ was constructed from the bulk wurtzite structure and fully relaxed to its lowest energy configuration. Subsequently, we replaced one, two, and four $\mathrm{Cd}(\mathrm{S})$ atoms with $\mathrm{S}(\mathrm{Cd})$ atoms in the outer shell layer to simulate $\mathrm{S}(\mathrm{Cd})$-rich QDs, and relaxed these structures to their lowest energy conformations. DFT calculations on larger cluster sizes, or on clusters with ligands, both of which better approximate the size of the measured QDs, would be desirable. However, in the current case the system of over 110 atoms is so large that computationally it is intractable to include significantly more atoms in the calculation. We recognize that for the $\mathrm{Cd}$ or $\mathrm{S}$ rich surfaces, maintaining charge neutrality without including ligands introduces additional reconstruction of surface atoms that may not be present on the larger QDs.

As could be seen from the projected density of states (PDOS) of Figure 3 for a stoichiometric cluster, the highest occupied molecular orbital (HOMO) is dominated by $\mathrm{S}$ atoms with very little contribution from Se atoms, while the lowest unoccupied molecular orbitals (LUMO) is dominated by Cd atoms. The charge density plots show that the HOMO and LUMO states are localized in the opposite surfaces of the cluster (Figure 3a). CdSe/CdS QDs are quasi-type-I particles, wherein the electron is relatively mobile throughout both core and shell, while the hole sees a much larger potential energy barrier at the $\mathrm{CdSe} / \mathrm{CdS}$ interface and, thus, is more localized in the core. A HOMO with significant contributions from the sulfur atoms in the shell is an interesting finding and presents a contrasting picture to this traditional particle-in-a-finite potential well model for a "core-shell" QD. Similar results are obtained for QDs with Cd-rich surfaces, although the HOMO-LUMO gap is reduced marginally (Figure 3). Narrowing of the gap 
is due to both decreased quantum confinement (as a result of the increased size of $\mathrm{Cd}$ atoms compared to $S$ atoms) and appearance of defect states near the edges of the valence and conduction bands.
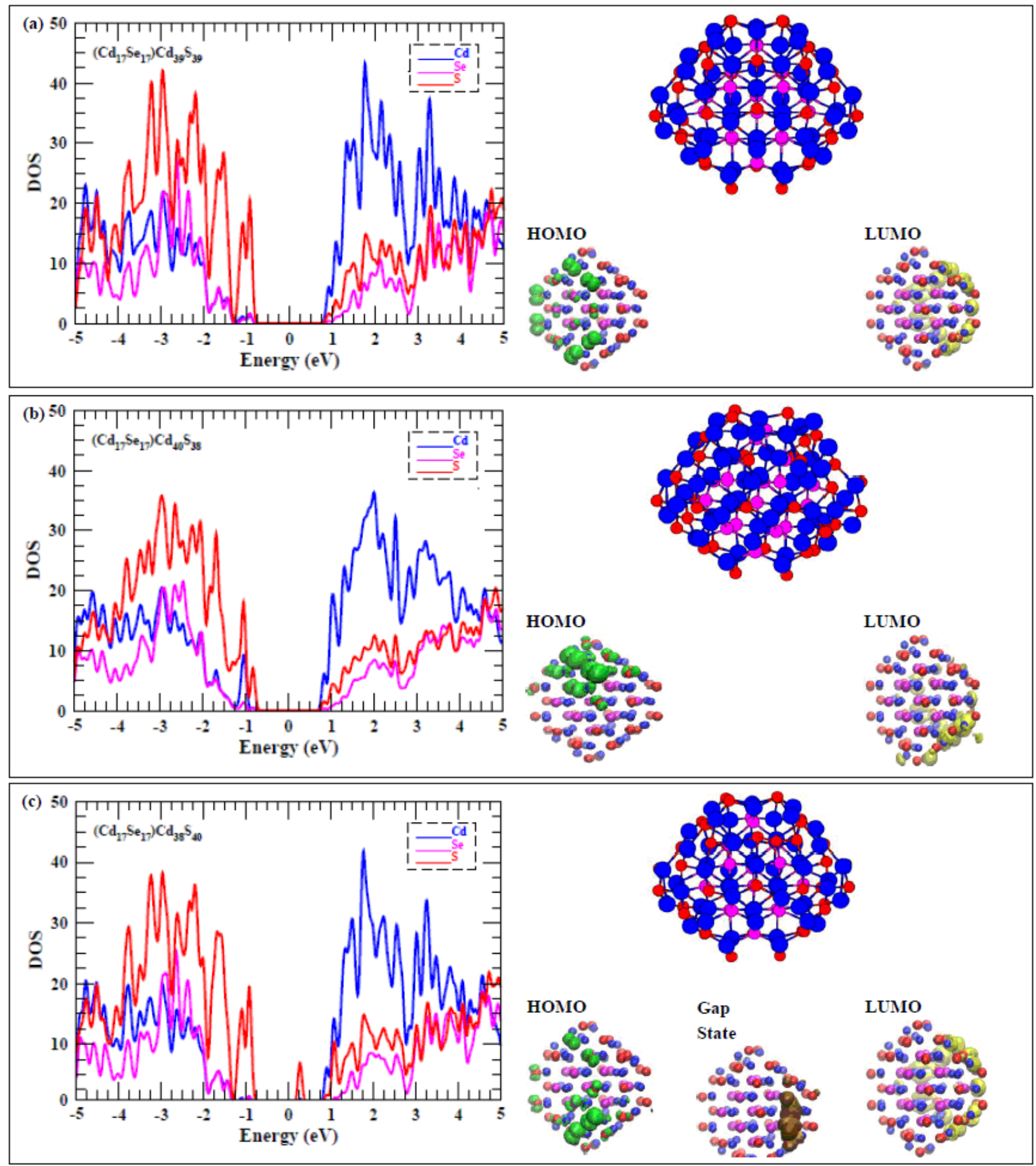

Figure3. (Left panel): Projected density of states (DOS) of $\mathrm{Cd}(\mathrm{blue}), \mathrm{Se}$ (magenta) and S(red) lines respectively of (a) stoichiometric $\left(\mathrm{Cd}_{17} \mathrm{Se}_{17}\right) \mathrm{Cd}_{39} \mathrm{~S}_{39}$ (b) Cd-rich $\left(\mathrm{Cd}_{17} \mathrm{Se}_{17}\right) \mathrm{Cd}_{40} \mathrm{~S}_{38}$ and (c) S-rich $\left(\mathrm{Cd}_{17} \mathrm{Se}_{17}\right) \mathrm{Cd}_{38} \mathrm{~S}_{40}$ core-shell QDs. The zero of energy is set equal to the energy of the middle of the 
HOMO-LUMO gap. (Top of right panel) The optimized geometry and [Bottom of right panel] charge density distribution of HOMO, LUMO and mid-gap states of the respective core-shell QDs. Blue, magenta and red balls represent $\mathrm{Cd}$, Se and $\mathrm{S}$ atoms, respectively.

The situation is qualitatively different in QDs with S-rich surfaces. The PDOS plot reveals appearance of localized defect states in the mid-gap region, $0.6 \mathrm{eV}$ below the conduction band edge (Figure 3c). Further increasing the concentration of surfaces $S$ atoms in the nonstoichiometric clusters increases the number and width of the mid-gap surface states, as shown in Figure S4.

To understand the origin of the mid-gap states in the sulfur-rich QDs, we analyzed the bonding patterns and Mulliken charge distribution of these nonstoichiometric clusters. Due to nonmetallic character, $\mathrm{S}$ atoms require formation of covalent bonds. In contrast, $\mathrm{Cd}$ atoms form metallic bonds that are less directional than covalent bonds and can accommodate stoichiometric defects much more easily. $\mathrm{S}$ atoms are more mobile on the QD surface, creating dangling bonds in S-rich nonstoichiometric QDs. Mulliken charge distributions reveal that the charges on the $S$ atoms responsible for the defects deviate from the charges of bulk $S$ atoms as well as from the charges of surface $S$ atoms in stoichiometric QDs. In particular, the defect $\mathrm{S}$ atoms are less anionic, indicating that chemical bonds are missing. On the other hand, even in highly nonstoichiometric Cd-rich QDs, structural reorganization prevents creation of dangling bonds.

The localized nature of the mid-gap states is particularly important and can play a crucial role in QD photophysical processes. Localized trap states can separate electrons and holes, causing a reduction in radiative decay rates. Further, localized states exhibit higher electron-phonon coupling ${ }^{66,67}$, which are shown to provide efficient nonradiative recombination pathways for electrons or holes ${ }^{67-69}$. Thus, we may predict that QDs with a higher S concentration in the shell layer efficiently quench fluorescence via both a reduction in the radiative rate (due to decreased electron-hole overlap) and an increased nonradiative recombination rate (due to mid-gap surface trap states).

Effect of Surface Composition on Fluorescence dynamics 
Photoluminescence decay dynamics were measured for CdSe core-only QDs and for CdSe/CdS core-shell QDs for each subsequent application of cadmium or sulfur to test these predictions. As expected, and consistent with similar systems ${ }^{70}$, the observed lifetimes were found to be multiexponential and comparable to literature values ${ }^{33}$. The multi-exponential nature of these ensemble measurements are indicative of surface trapping and recombination on many timescales ${ }^{71}$, as opposed to single exponential data, which signifies high quality, high quantum yield QDs with near-perfect surfaces.

Several interesting trends are observed in the PL lifetime data as subsequent layers of $\mathrm{Cd}$ or $\mathrm{S}$ are applied. In general, as the CdS shell grows larger, the PL lifetime increases (Figure 4). This effect has been observed previously and attributed to reduced electron-hole overlap in the CdSe/CdS QD due to the electron delocalizing over the entire nanoparticle ${ }^{33}$. Upon application of each successive sulfur layer, the PL lifetime shortens compared to the previous cadmium terminated value (Figure 4). This decrease in PL lifetime is accompanied by a drop in PL QY (Table S2). The opposite effect occurs with application of each successive Cd layer: the PL lifetime gets longer which is accompanied by an increase in PL QY (Table S2). We note that the application of the first Cd layer on a CdSe core QD (forming CdSe/Cd QDs) does not follow the overall trend seen as the CdS shell gets thicker. In general application of the first Cdshell led to a small change in QY (Figure 2), with a proportional drop in the PL lifetime (Figure 4). Thus, the change in QY is almost exclusively due to an increase in the non-radiative rate with the application of an additional $\mathrm{Cd}$ layer. As discussed in the previous theoretical section, the increase in non-radiative rate could arise from an increase in defect states near the conduction and valence band edges.

Using the definition of $Q Y, Q Y=k_{r} /\left(k_{r}+k_{n r}\right)$, the impact of surface composition on the QD's radiative and nonradiative recombination rates can be characterized (Figure 4d). The drop in QY in sulfur terminated QDs is due to both an increase in the non-radiative rate and a decrease in the radiative rate (compared to cadmium terminated surfaces). Both effects are consistent with the predictions of the 
DFTB calcuations. The nonradiative rate increase originates from the increase in the number mid-gap hole states. Similar studies have found comparable patterns in CdTe nanoparticles with increasing telluride concentration on surfaces ${ }^{19}$, where hole traps and less passivated telluride surfaces contribute to increased nonradiative rates. Further, selenium rich surfaces on CdSe cores also produce insufficiently passivated particle surfaces ${ }^{54}$ and comparable changes in fluorescent lifetimes ${ }^{21}$. The radiative rate decrease is consistent with localization of holes on surface sulfur atoms, which causes increased electron-hole separation. The PL lifetime data for cadmium terminated QDs is also consistent with the DFTB calculations. Although the DFTB calculations indicate the LUMO wavefunctions are localized on the Cd atoms, Cd-termination does not lead to a high density of surface trap states and only a weak sensitivity of $k_{r}$ and $k_{n r}$ to the QD size are observed. Thus, we conclude that the mid-gap trap states (which are only observed in the sulfur terminated particles) are critical in mediating the PL QY of the core/shell particles. 

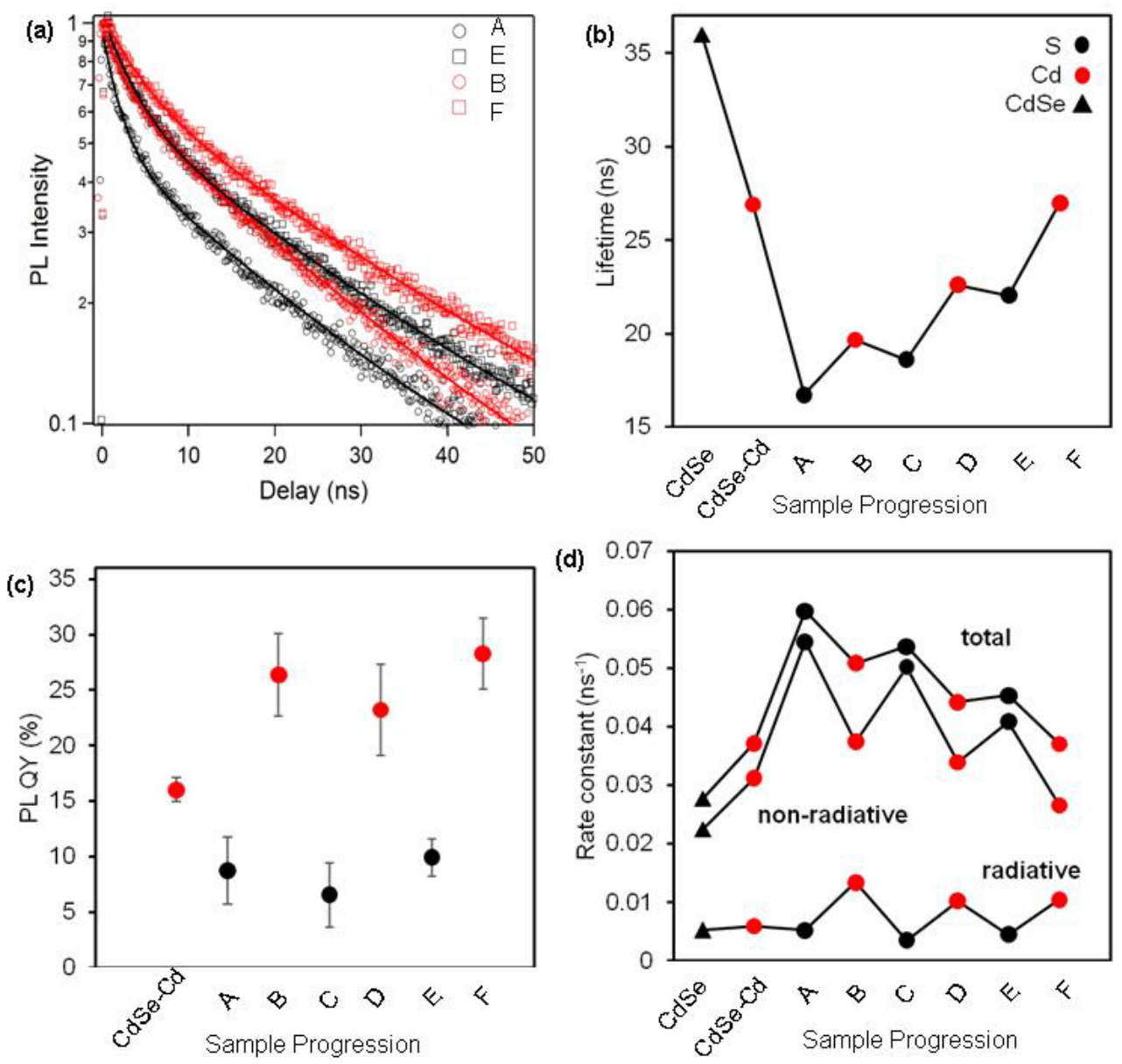

Figure 4. PL decay dynamics. Lines and symbols in red indicate cadmium-terminated QDs, whereas black indicates sulfur-terminated QDs, or the seed CdSe core where indicated. (a) Lifetime decay curves for select samples shown in Figure 2. Samples $A$ and $E$ denote full shells, thus sulfur terminated. Samples $B$ and $F$ denote the subsequent applications of cadmium, thus cadmium terminated. (b) Amplitude weighted lifetimes as a function of shell growth. (c) Average PL quantum yields for each shell. (d) Radiative and non-radiative rate constants as a function of cadmium or sulfur termination.

\section{Conclusions}

Seed CdSe/CdS QDs were synthesized using secondary phosphine chalcogenide and cadmium carboxylate precursors using a SILAR-type method. This method allowed for well-controlled surface compositions that can be continually tuned from predominantly cadmium to predominantly sulfur termination. As seen in CdSe and CdS QDs, surface composition correlates with sample fluorescence 
intensity: chalcogen termination quenches PL intensity while metal termination restores QD PL. This surface termination photophysical effect is propagated into lifetime data, where nonradiative decay components drastically increase and dominate lifetimes, especially of sulfur terminated particles. DFTB analysis indicates the presence of midgap states arising from sulfur termination, in agreement with previous studies of bare cores. Despite the quasi-type-I electronic heterostructure of these core/shell particles, these midgap states are shown by DFTB to act as hole traps on the surface, similarly to CdS core QDs. In conjunction with the theory, the drastic and reversible quenching of fluorescence on chalcogen terminated samples suggests that CdS is not an effective hole-confining shell for CdSe, as surface hole traps remain accessible in spite of the shell.

A notable implication of these findings is that sulfur terminated samples may provide more direct access to surface holes than their cadmium terminated counterparts. The inefficiencies of CdS as a hole confining shell, combined with the accessible hole trap states on the surface, make CdSe/CdS systems with sulfur termination viable candidates for QD-sensitized photocatalytic hydrogen generation systems ${ }^{9,10,72}$, where hole quenching in QDs by a sacrificial donor has been shown to be the rate limiting step $^{73}$.

\section{Acknowledgements}

This work was supported by the National Science Foundation through CHE- 1307254: (B.S., T.D.K., K.S., J.P.) and CHE-1300118 (O.P.) and the Department of Energy Office of Basic Energy Sciences through Grant DE-FG02-06ER15821 (Z. H.).

\section{References and Notes}

1. A. I. Ekimov and A. A. Onushchenko, Pis'ma Zh. Eksp. Teor. Fiz. 34, 363-366 (1981).

2. A. P. Alivisatos, Science 271 (5251), 933-937 (1996).

3. R. Rossetti, S. Nakahara and L. E. Brus, J. Chem. Phys. 79, 1086-1088 (1983).

4. C. M. Evans, L. Guo, J. J. Peterson, S. Maccagnano-Zacher and T. D. Krauss, Nano Lett. 8 (9), 2896 (2008). 
5. M. Nirmal, B. O. Dabbousi, M. G. Bawendi, J. J. Macklin, J. K. Trautman, T. D. Harris and L. E. Brus, Nature 383 (6603), 802-804 (1996).

6. M. T. Frederick and E. A. Weiss, ACS Nano 4 (6), 3195-3200 (2010).

7. S. Jin, R. D. Harris, B. Lau, K. O. Aruda, V. A. Amin and E. A. Weiss, Nano Letters 14 (9), 5323-5328 (2014).

8. C. B. Murray, C. R. Kagan and M. G. Bawendi, Annual Review of Materials Science 30 (1), 545610 (2000).

9. Z. Han, F. Qiu, R. Eisenberg, P. L. Holland and T. D. Krauss, Science 338 (6112), 1321-1324 (2012).

10. K. Wu, H. Zhu and T. Lian, Accounts of Chemical Research 48 (3), 851-859 (2015).

11. D. S. Ginger and N. C. Greenham, Phys. Rev. B 59, 10622 (1999).

12. I. Robel, V. Subramanian, M. Kuno and P. V. Kamat, Journal of the American Chemical Society 128 (7), 2385-2393 (2006).

13. S. Coe, W.-K. Woo, M. Bawendi and V. Bulovic, Nature 420 (6917), 800-803 (2002).

14. S. Coe-Sullivan, W.-K. Woo, J. S. Steckel, M. Bawendi and V. Bulović, Organic Electronics 4 (2-3), $123-130$ (2003).

15. J. K. Jaiswal, H. Mattoussi, J. M. Mauro and S. M. Simon, Nat Biotech 21 (1), 47-51 (2003).

16. M. Bruchez, Jr., M. Moronne, P. Gin, S. Weiss and A. P. Alivisatos, Science 281 (5385), 2013-2016 (1998).

17. T. C. Pappas, W. M. S. Wickramanyake, E. Jan, M. Motamedi, M. Brodwick and N. A. Kotov, Nano Letters 7 (2), 513-519 (2007).

18. V. V. Breus, C. D. Heyes and G. U. Nienhaus, The Journal of Physical Chemistry C 111 (50), 1858918594 (2007).

19. B. Omogo, J. F. Aldana and C. D. Heyes, The Journal of Physical Chemistry C 117 (5), 2317-2327 (2013).

20. K. B. Subila, G. K. Kumar, S. M. Shivaprasad and K. G. Thomas, J. Phys. Chem. Lett. 4, 2774 (2013).

21. Y. Gao and X. Peng, Journal of the American Chemical Society 137 (12), 4230-4235 (2015).

22. O. Chen, Y. Yang, T. Wang, H. Wu, C. Niu, J. Yang and Y. C. Cao, Journal of the American Chemical Society 133 (43), 17504-17512 (2011).

23. M. Kuno, J. K. Lee, B. O. Dabbousi, F. V. Mikulec and M. G. Bawendi, The Journal of Chemical Physics 106 (23), 9869-9882 (1997).

24. M. A. Hines and P. Guyot-Sionnest, J. Phys. Chem. 100 (2), 468 (1996).

25. J. S. Steckel, J. P. Zimmer, S. Coe-Sullivan, N. E. Stott, V. Bulović and M. G. Bawendi, Angewandte Chemie International Edition 43 (16), 2154-2158 (2004).

26. V. V. Protasenko, M. Kuno, A. Gallagher and D. J. Nesbitt, Optics Communications 210 (1-2), 1123 (2002).

27. C. B. Murray, D. J. Norris and M. G. Bawendi, J. Am. Chem. Soc. 115, 8706-8715 (1993).

28. B. O. Dabbousi, J. Rodriguez-Viejo, F. V. Mikulec, J. R. Heine, H. Mattoussi, R. Ober, K. F. Jensen and M. G. Bawendi, The Journal of Physical Chemistry B 101 (46), 9463-9475 (1997).

29. G. E. Cragg and A. L. Efros, Nano Letters 10 (1), 313-317 (2010).

30. Q. Lin, N. S. Makarov, W.-k. Koh, K. A. Velizhanin, C. M. Cirloganu, H. Luo, V. I. Klimov and J. M. Pietryga, ACS Nano 9 (1), 539-547 (2015).

31. Y. Chen, J. Vela, H. Htoon, J. L. Casson, D. J. Werder, D. A. Bussian, V. I. Klimov and J. A. Hollingsworth, Journal of the American Chemical Society 130 (15), 5026-5027 (2008).

32. B. Mahler, N. Lequeux and B. Dubertret, Journal of the American Chemical Society 132 (3), $953-$ 959 (2010).

33. W. K. Bae, L. A. Padilha, Y.-S. Park, H. McDaniel, I. Robel, J. M. Pietryga and V. I. Klimov, ACS Nano 7 (4), 3411-3419 (2013). 
34. A. M. Smith, L. A. Lane and S. Nie, Nat Commun 5 (2014).

35. X. Zhong, M. Han, Z. Dong, T. J. White and W. Knoll, Journal of the American Chemical Society 125 (28), 8589-8594 (2003).

36. H. Zhu and T. Lian, Energy \& Environmental Science 5 (11), 9406-9418 (2012).

37. S. Kim, B. Fisher, H.-J. Eisler and M. Bawendi, Journal of the American Chemical Society 125 (38), 11466-11467 (2003).

38. S. A. Ivanov, A. Piryatinski, J. Nanda, S. Tretiak, K. R. Zavadil, W. O. Wallace, D. Werder and V. I. Klimov, Journal of the American Chemical Society 129 (38), 11708-11719 (2007).

39. M. V. Kovalenko, M. I. Bodnarchuk, J. Zaumseil, J.-S. Lee and D. V. Talapin, Journal of the American Chemical Society 132 (29), 10085-10092 (2010).

40. N. C. Anderson, M. P. Hendricks, J. J. Choi and J. S. Owen, Journal of the American Chemical Society 135 (49), 18536-18548 (2013).

41. C. M. Evans, M. E. Evans and T. D. Krauss, Journal of the American Chemical Society 132 (32), 10973-10975 (2010).

42. J. S. Steckel, B. K. H. Yen, D. C. Oertel and M. G. Bawendi, Journal of the American Chemical Society 128 (40), 13032-13033 (2006).

43. H. H.-Y. Wei, C. M. Evans, B. D. Swartz, A. J. Neukirch, J. Young, O. V. Prezhdo and T. D. Krauss, Nano Letters 12 (9), 4465-4471 (2012).

44. N. J. Borys, M. J. Walter, J. Huang, D. V. Talapin and J. M. Lupton, Science 330 (6009), 1371-1374 (2010).

45. D. Steiner, D. Dorfs, U. Banin, F. Della Sala, L. Manna and O. Millo, Nano Letters 8 (9), 2954-2958 (2008).

46. A. Pandey and P. Guyot-Sionnest, The Journal of Chemical Physics 127 (10), 104710 (2007).

47. J. J. Li, Y. A. Wang, W. Guo, J. C. Keay, T. D. Mishima, M. B. Johnson and X. Peng, Journal of the American Chemical Society 125 (41), 12567-12575 (2003).

48. J. Jasieniak and P. Mulvaney, Journal of the American Chemical Society 129 (10), 2841-2848 (2007).

49. W. W. Yu and X. Peng, Angewandte Chemie International Edition 41 (13), 2368-2371 (2002).

50. W. W. Yu, L. Qu, W. Guo and X. Peng, Chemistry of Materials 15 (14), 2854-2860 (2003).

51. C. D. Wagner, W. M. Riggs, L. E. Davis, J. F. Moulder and G. E. Muilenberg, Handbook of X-ray Photoelectron Spectroscopy. (Physical Electronics, Inc., 1978).

52. A. R. West, Basic Solid State Chemistry. (1988).

53. J. Nanda, B. A. Kuruvilla and D. D. Sarma, Phys. Rev. B 59, 7473 (1999).

54. J. E. B. Katari, V. L. Colvin and A. P. Alivisatos, J. Phys. Chem. 98, 4109 (1994).

55. D. Porezag, T. Frauenheim, T. Köhler, G. Seifert and R. Kaschner, Physical Review B 51 (19), 12947-12957 (1995).

56. M. Elstner, D. Porezag, G. Jungnickel, J. Elsner, M. Haugk, T. Frauenheim, S. Suhai and G. Seifert, Physical Review B 58 (11), 7260-7268 (1998).

57. T. A. Niehaus, S. Suhai, F. Della Sala, P. Lugli, M. Elstner, G. Seifert and T. Frauenheim, Physical Review B 63 (8), 085108 (2001).

58. G. Seifert, The Journal of Physical Chemistry A 111 (26), 5609-5613 (2007).

59. S. Sarkar, S. Pal, P. Sarkar, A. L. Rosa and T. Frauenheim, Journal of Chemical Theory and Computation 7 (7), 2262-2276 (2011).

60. M. S. Setty and A. P. B. Sinha, Thin Solid Films 144 (1), 7-19 (1986).

61. S. Tanuma, C. J. Powell and D. R. Penn, Surface and Interface Analysis 20 (1), 77-89 (1993).

62. S. Tanuma, C. J. Powell and D. R. Penn, Surface and Interface Analysis 21 (3), 165-176 (1994).

63. Y. Liu, M. Kim, Y. Wang, Y. A. Wang and X. Peng, Langmuir 22 (14), 6341-6345 (2006). 
64. S. F. Wuister, C. de Mello Donegá and A. Meijerink, The Journal of Physical Chemistry B 108 (45), 17393-17397 (2004).

65. O. Chen, J. Zhao, V. P. Chauhan, J. Cui, C. Wong, D. K. Harris, H. Wei, H.-S. Han, D. Fukumura, R. K. Jain and M. G. Bawendi, Nat Mater 12 (5), 445-451 (2013).

66. B. F. Habenicht, H. Kamisaka, K. Yamashita and O. V. Prezhdo, Nano Letters 7 (11), 3260-3265 (2007).

67. B. F. Habenicht, C. F. Craig and O. V. Prezhdo, Physical Review Letters 96 (18), 187401 (2006).

68. B. F. Habenicht, O. N. Kalugin and O. V. Prezhdo, Nano Letters 8 (8), 2510-2516 (2008).

69. B. F. Habenicht and O. V. Prezhdo, The Journal of Physical Chemistry C 113 (32), 14067-14070 (2009).

70. A. V. Malko, Y.-S. Park, S. Sampat, C. Galland, J. Vela, Y. Chen, J. A. Hollingsworth, V. I. Klimov and $\mathrm{H}$. Htoon, Nano Letters 11 (12), 5213-5218 (2011).

71. K. E. Knowles, E. A. McArthur and E. A. Weiss, ACS Nano 5 (3), 2026-2035 (2011).

72. A. Vaneski, J. Schneider, A. S. Susha and A. L. Rogach, APL Materials 2 (1), 012104 (2014).

73. K. Wu, Z. Chen, H. Lv, H. Zhu, C. L. Hill and T. Lian, Journal of the American Chemical Society 136 (21), 7708-7716 (2014). 